\title{
Hidden symmetries of two-electron quantum dots in a magnetic field
}

\author{
N. S. Simonović ${ }^{1}$ and R.G. Nazmitdinov ${ }^{1,2,3}$ \\ ${ }^{1}$ Max-Planck-Institut für Physik komplexer Systeme, D-01187 Dresden, Germany \\ 2 Departament de Física, Universitat de les Illes Balears, E-07071 Palma de Mallorca, Spain \\ ${ }^{3}$ Bogoliubov Laboratory of Theoretical Physics, Joint Institute for Nuclear Research, 141980 Dubna, Russia
}

\begin{abstract}
Using a classical and quantum mechanical analysis, we show that the magnetic field gives rise to dynamical symmetries of a three-dimensional axially symmetric two-electron quantum dot with a parabolic confinement. These symmetries manifest themselves as near-degeneracies in the quantum spectrum at specific values of the magnetic field and are robust at any strength of the electron-electron interaction.
\end{abstract}

PACS number(s): 03.65.Ge, 73.21.La, 05.45.Mt, $75.75 .+\mathrm{a}$

A three-dimensional harmonic oscillator with frequencies in rational ratios $(\mathrm{RHO})$ and a Coulomb system are benchmarks for the hidden symmetries which account for the accidental degeneracies of their quantum spectra (see, e.g., [1]). Recent advances in nanotechnology create a remarkable opportunity to trace their dynamical interplay in mesoscopic systems like quantum dots, which confine a few electrons to a space of a few hundred nanometers. Indeed, competition between a confining potential, approximated quite well by the harmonic oscillator $(\mathrm{HO})$, and the repulsive electron-electron interaction produces a rich variety of phenomena, for example, in a two-electron quantum dot (QD) under a perpendicular magnetic field (see [3,2] and references therein). In fact, a two-electron QD becomes a testing-ground for different quantum-mechanical approaches [2] and experimental techniques that could provide highly accurate data for this system $[4,3]$.

If the $\mathrm{HO}$ and the Coulomb potential are combined, most of the symmetries are expected to be broken. Nevertheless, in particular cases, the Coulomb (Kepler) sys- tem and the RHO may have common symmetries, as it was already noticed a long time ago [5]. The authors of Ref. [5] could not find, however, a physical application for this phenomenon. These symmetries were rediscovered in the analysis of laser-cooled ions in a Paul trap [6] and of the hydrogen atom in the generalized van der Waals potential [7]. The major aspect of the present paper is to demonstrate that the hidden symmetries could be observed in a two-electron QD under a tunable perpendicular magnetic field if the effective confinement potential is indeed the three-dimensional (3D) HO. To this aim we focus our analysis upon the nonlinear classical dynamics of the system. At certain conditions the motion becomes integrable and this indicates the existence of the symmetries in the quantum spectrum.

The Hamiltonian of a two-electron QD in a magnetic field reads

$$
H=\sum_{j=1}^{2}\left[\frac{1}{2 m^{*}}\left(\mathbf{p}_{j}-\frac{e}{c} \mathbf{A}_{j}\right)^{2}+U\left(\mathbf{r}_{j}\right)\right]+\frac{\alpha}{\left|\mathbf{r}_{1}-\mathbf{r}_{2}\right|}
$$

where $\alpha=e^{2} / 4 \pi \varepsilon_{0} \varepsilon_{r}$. Here, $e, m^{*}, \varepsilon_{0}$ and $\varepsilon_{r}$ are the unit charge, effective electron mass, vacuum and relative dielectric constants of a semiconductor, respectively. For the perpendicular magnetic field we choose the vector potential with a gauge $\mathbf{A}=\frac{1}{2} \mathbf{B} \times \mathbf{r}=\frac{1}{2} B(-y, x, 0)$. The confining potential is approximated by a 3D axiallysymmetric HO $U(\mathbf{r})=m^{*}\left[\omega_{0}^{2}\left(x^{2}+y^{2}\right)+\omega_{z}^{2} z^{2}\right] / 2$, where $\hbar \omega_{z}$ and $\hbar \omega_{0}$ are the energy scales of confinement in the $z$-direction and in the $x y$-plane, respectively. In contrast to a $2 \mathrm{D}$ description of the $\mathrm{QD}$ this approximation pro- 
vides a consistent description [8] of various experimental features [9]: the energy spectrum for small magnetic field, the value of the magnetic field for the first singlet-triplet transition, and the ratio of the lateral to vertical extension of the dot. In the present analysis we neglect the spin interaction, since the corresponding energy is small compared to the confinement and the Coulomb energies. Introducing the relative and center-of-mass (CM) coordinates $\mathbf{r}=\mathbf{r}_{1}-\mathbf{r}_{2}, \mathbf{R}=\frac{1}{2}\left(\mathbf{r}_{1}+\mathbf{r}_{2}\right)$, the Hamiltonian (1) is separated into the CM and relative-motion terms $H=H_{\mathrm{CM}}+H_{\text {rel }}[10]$. The solution to the first term is well known [11]. It possesses all symmetries of the $\mathrm{HO}$, since the Coulomb interaction enters the second term for the parabolic confinement [12]. In the following we concentrate on the dynamics of $H_{\text {rel }}$. For our analysis it is convenient to use cylindrical scaled coordinates, $\tilde{\rho}=\rho / l_{0}, \tilde{p}_{\rho}=p_{\rho} l_{0} / \hbar, \tilde{z}=z / l_{0}, \tilde{p}_{z}=p_{z} l_{0} / \hbar$, where $l_{0}=\left(\hbar / \mu \omega_{0}\right)^{1 / 2}$ is the characteristic length of the confinement potential with the reduced mass $\mu=m^{*} / 2$. The strength parameter $\alpha$ of the Coulomb repulsion goes over to $\lambda=2 \alpha /\left(\hbar \omega_{0} l_{0}\right)$. Although our consideration is general, for the demonstration we choose the values $\hbar \omega_{0} \approx 2.8 \mathrm{meV}$ and $\omega_{z}=2.5 \omega_{0}$ which are close to those obtained in our 3D analysis [8] of the experiment [9]. For the effective mass $m^{*}=0.067 m_{e}$ and the dielectric constant $\varepsilon=12$, which are typical for GaAs, the value $\lambda=3$. Hereafter, for the sake of simplicity, we drop the tilde, i.e. for the scaled variables we use the same symbols as before scaling.

In these variables the Hamiltonian for the relative motion takes the form (in units of $\hbar \omega_{0}$ )

$h \equiv \frac{H_{\mathrm{rel}}}{\hbar \omega_{0}}=\frac{1}{2}\left(p_{\rho}^{2}+\frac{m^{2}}{\rho^{2}}+p_{z}^{2}+\tilde{\omega}_{\rho}^{2} \rho^{2}+\tilde{\omega}_{z}^{2} z^{2}+\frac{\lambda}{r}\right)-\tilde{\omega}_{L} m$ where $r=\left(\rho^{2}+z^{2}\right)^{1 / 2}, \tilde{\omega} \equiv \omega / \omega_{0}, m=l_{z} / \hbar$. Here, $\omega_{L}=$ $e B / 2 m^{*} c$ is the Larmor frequency and $\omega_{\rho}=\left(\omega_{L}^{2}+\omega_{0}^{2}\right)^{1 / 2}$ is the effective confinement frequency in the $\rho$-coordinate, which depends through $\omega_{L}$ on the magnetic field. In this way the magnetic field can be used to control the effective lateral confinement frequency of the QD for a fixed value of the vertical confinement, i.e. the ratio $\omega_{z} / \omega_{\rho}$.

Beside the energy $(\epsilon \equiv h)$, the $z$-component of angular momentum $l_{z}$ is also an integral of motion due to the axial symmetry of the system. Therefore, the magnetic quantum number $m$ is always a good quantum number. Since the Hamiltonian (2) is invariant under the reflection of the origin, the parity $\pi$ is a good quantum number too.

Although the motion in $\varphi$ is separated from the motion in the $(\rho, z)$-plane, the problem is in general nonintegrable, since the Coulomb term couples the $\rho$ and $z$-coordinates. Examination of the Poincaré sections by varying the parameter $\omega_{z} / \omega_{\rho}$ (see Fig. 1 for examples) in the interval $(1 / 10,10)$ with a small step indicates that there are five integrable cases. The trivial cases are $\omega_{z} / \omega_{\rho} \rightarrow 0$ and $\omega_{z} / \omega_{\rho} \rightarrow \infty$, which correspond to $1 \mathrm{D}$ circular and 2D vertical QDs, respectively. The nontrivial cases are $\omega_{z} / \omega_{\rho}=1 / 2,1,2$. These results hold for any strength of the Coulomb interaction and agree with the results for the Paul trap [6]. Below we discuss the nontrivial cases only. The typical trajectories in cylindrical coordinates are shown in Figs. 2a,c.

The results obtained with the aid of the Poincaré surfaces of sections are invariant under the coordinate transformation. On the other hand, the integrability is a necessary condition for the existence of a coordinate system in which the motion can be separated. In turn, the analogous quantum mechanical system would be characterized 
by a complete set of quantum numbers.

At the value $\omega_{L}^{\prime}=\left(\omega_{z}^{2}-\omega_{0}^{2}\right)^{1 / 2}$ the magnetic field gives rise to a spherical symmetry $\left(\omega_{z} / \omega_{\rho}=1\right)$ in the axially symmetric QD [8]. In this case the Hamiltonian (2) is separable in (scaled) spherical coordinates (see Eq. (7) in [8]). The additional integral of motion is the square of the total angular momentum $\mathbf{l}^{2}$. The spherical coordinates are a particular limit of the spheroidal (elliptic) coordinates well suitable for the analysis of the Coulomb systems (see, e.g., [13]). Therefore, to search the separability for the other integrable cases it is convenient to use the spheroidal coordinates $(\xi, \eta, \varphi)$, where $\xi=\left(r_{1}+r_{2}\right) / d$ and $\eta=\left(r_{1}-r_{2}\right) / d$. In the prolate spheroidal coordinates $r_{1}=\left[\rho^{2}+(z+d)^{2}\right]^{1 / 2}, r_{2}=r$. The parameter $d \in(0, \infty)$ is the distance between two foci of the coordinate system (with the origin at one of them). In the limit $d \rightarrow 0$ the motion is separated when $\omega_{z} / \omega_{\rho}=1$ (Fig. 2b). In this limit $\xi \rightarrow \infty$ such that $r=d \xi / 2$ is finite, $\eta=\cos \vartheta$, and we obtain the spherical coordinate system.

Let us turn to the case $\omega_{z} / \omega_{\rho}=2$ which occurs at the value of the magnetic field $\omega_{L}^{\prime \prime}=\left(\omega_{z}^{2} / 4-\omega_{0}^{2}\right)^{1 / 2}$. In the prolate spheroidal coordinates the motion is separated in the limit $d \rightarrow \infty$ (Fig. 2d). In fact, at $d \rightarrow \infty: \xi \rightarrow 1$, $\eta \rightarrow 1$ such that $\xi_{1}=d(\xi-1), \xi_{2}=d(1-\eta)$ are finite, - we obtain the parabolic coordinate system $\left(\xi_{1}, \xi_{2}, \varphi\right)$ where $\xi_{1,2}=r \pm z$. In these coordinates the Hamiltonian (2) has the form

$$
\begin{aligned}
h & =\frac{1}{\xi_{1}+\xi_{2}}\left[2\left(\xi_{1} p_{\xi_{1}}^{2}+\xi_{2} p_{\xi_{2}}^{2}\right)+\frac{m^{2}}{2}\left(\frac{1}{\xi_{1}}+\frac{1}{\xi_{2}}\right)\right. \\
& \left.+\frac{\tilde{\omega}_{z}^{2}}{8}\left(\xi_{1}^{3}+\xi_{2}^{3}\right)+\lambda\right]-\tilde{\omega}_{L}^{\prime \prime} m
\end{aligned}
$$

and the equation $\left(\xi_{1}+\xi_{2}\right)(h-\epsilon)=0$ is separated into two decoupled equations for $\xi_{1}$ and $\xi_{2}$ variables. Simple manipulations define the separation constant

$$
c=a_{z}-\tilde{\omega}_{\rho}^{2} \rho^{2} z
$$

which is a desired third integral of motion. Here $a_{z}$ is the z-component of the Runge-Lenz vector $\mathbf{a}=\mathbf{p} \times$ $\mathbf{l}+\lambda \mathbf{r} /(2 r)$, which is a constant of motion for the pure Coulomb system (i.e. when $\omega_{\rho}=\omega_{z}=0$ ) [1]. The quantum mechanical counterpart of the integral of motion, Eq. (4), does not commute with the parity operator and we should expect the degeneracy of quantum levels.

Due to the separability of the motion in the parabolic coordinate system, the eigenfunctions of the corresponding Schrödinger equation can be expressed in the form $\psi(\mathbf{r})=f_{1}\left(\xi_{1}\right) f_{2}\left(\xi_{2}\right) e^{i m \varphi}$, where the functions $f_{j}$ are solutions of the equations

$$
\begin{aligned}
\frac{d}{d \xi_{j}}\left(\xi_{j} \frac{d f_{j}}{d \xi_{j}}\right) & -\frac{1}{4}\left[\frac{m^{2}}{\xi_{j}}+\frac{\tilde{\omega}_{z}^{2}}{4} \xi_{j}^{3}-2\left(\epsilon+\tilde{\omega}_{L}^{\prime \prime} m\right) \xi_{j}\right. \\
& \left.+\lambda-(-1)^{j} 2 c\right] f_{j}=0, \quad j=1,2 .
\end{aligned}
$$

Eqs. (5) can be solved numerically and the eigenenergies and eigenvalues of $c$ are determined iteratively by varying simultaneously $\epsilon$ and $c$ until the functions $f_{j}$ fulfill the boundary conditions: $f_{j} \sim \xi_{j}^{|m| / 2}, \xi_{j} d f_{j} / d \xi_{j}=|m| f_{j} / 2$ for $\xi_{j} \rightarrow 0$ and $f_{j} \rightarrow 0$ for $\xi_{j} \rightarrow \infty$. Let $n_{1}$ and $n_{2}$ be the nodal quantum numbers of the functions $f_{1}$ and $f_{2}$, respectively. Note that Eqs. (5) are coupled by the constants of motion and, therefore, both functions depend on all three quantum numbers $\left(n_{1}, n_{2}, m\right)$. However, the simple product of these functions have no a definite parity. Since $\mathbf{r} \rightarrow-\mathbf{r} \Leftrightarrow\left\{\xi_{1} \rightarrow \xi_{2}, \xi_{2} \rightarrow \xi_{1}, \varphi \rightarrow \varphi+\pi\right\}$, the even/odd eigenfunctions are constructed as

$$
\begin{aligned}
\psi_{N, k, m}^{( \pm)}(\mathbf{r}) & =\frac{e^{i m \varphi}}{\sqrt{2}}\left[f_{n_{1}}^{\left(n_{2}, m\right)}\left(\xi_{1}\right) f_{n_{2}}^{\left(n_{1}, m\right)}\left(\xi_{2}\right)\right. \\
& \left. \pm(-1)^{m} f_{n_{2}}^{\left(n_{1}, m\right)}\left(\xi_{1}\right) f_{n_{1}}^{\left(n_{2}, m\right)}\left(\xi_{2}\right)\right]
\end{aligned}
$$

where $N=n_{1}+n_{2}$ and $k=\left|n_{1}-n_{2}\right|$. These states are the eigenfunctions of $h, l_{z},|c|$ and the parity operator. For 
$|c|>0$ the eigenstates (6) appear in doublets of different parity and, therefore, of a different total spin. For $c=0$ in Eqs. (5) $f_{1}=f_{2}$ and, obviously, only the states with parity $\pi=(-1)^{m}$ exist.

For the magnetic field $\omega_{L}^{\prime \prime \prime} \equiv\left(4 \omega_{z}^{2}-\omega_{0}^{2}\right)^{1 / 2}$ we obtain the ratio $\omega_{z} / \omega_{\rho}=1 / 2$. The Hamiltonian (2) expressed in the oblate spheroidal coordinates $\left(r_{1}=\left[z^{2}+(\rho+d)^{2}\right]^{1 / 2}\right.$, $\left.r_{2}=r\right)$ is separated for $m=0($ at $d \rightarrow \infty)$. For $m \neq 0$ the term $m^{2} / \rho^{2}$ and, consequently, the Hamiltonian (2) is not separated in these coordinates. Note, for $m=0$ the cases $\omega_{z} / \omega_{\rho}=1 / 2$ and 2 are equivalent if we exchange the $\rho$ and $z$ coordinates and, hence, the additional integral of motion is $\left|a_{\rho}-\tilde{\omega}_{z}^{2} z^{2} \rho\right|$. For $m \neq 0$ we use the procedure described in Ref. [6] and obtain the following integral of motion

$$
C=\left[\left(a_{\rho}-\tilde{\omega}_{z}^{2} z^{2} \rho\right)^{2}+a_{\varphi}^{2}+4 m^{2} \tilde{\omega}_{z}^{2} r^{2}\right]^{1 / 2},
$$

where $a_{\rho}$ and $a_{\varphi}$ are the $\rho$ and $\varphi$ components of the Runge-Lenz vector, respectively. Due to existence of three independent integrals of motion, $h, m$ and $c$, which are in involution, the dynamics for $m \neq 0$, although nonseparable, is integrable. The further analysis for $m=0$ is similar to the previous one and we omit it here.

Let us denote by $h^{*}$ the Hamiltonian (2) for a specific value of the magnetic field when the system becomes separable, i.e. for $\omega_{L}^{*}=\omega_{L}^{\prime}, \omega_{L}^{\prime \prime}$ or $\omega_{L}^{\prime \prime \prime}$ (for $m=0$ ). Then for an arbitrary value of $\omega_{L}$ we can write

$$
h=h^{*}+\frac{1}{2} \Delta \tilde{\omega}_{L}^{2} \rho^{2}-\Delta \tilde{\omega}_{L} m,
$$

where $\Delta \tilde{\omega}_{L}^{2}=\tilde{\omega}_{L}^{2}-\tilde{\omega}_{L}^{* 2}, \Delta \tilde{\omega}_{L}=\tilde{\omega}_{L}-\tilde{\omega}_{L}^{*}$ and the term $\frac{1}{2} \Delta \tilde{\omega}_{L}^{2} \rho^{2}$ is the only non-diagonal part of $h$ in the eigenbasis of $h^{*}$. The eigenenergies of the Hamiltonian (2) (see Fig. 3) have been calculated with the use of the basis (6) and the spherical basis in the intervals $0 \leq \tilde{\omega}_{L} \leq 1.5$ and the $1.5 \leq \tilde{\omega}_{L} \leq 5$, respectively. The radial parts of the spherical eigenfunctions and $f_{n_{1}, n_{2}, m}$, as well as the corresponding factors in the matrix elements $\left\langle\psi_{i}\left|\rho^{2}\right| \psi_{j}\right\rangle$, are evaluated numerically. The complete spectrum of the two-electron QD (Fig. 3a) shows the accumulation of levels with different quantum numbers into well pronounced bands at strong magnetic field. There is no obvious manifestation of the symmetries discussed above. In fact, the effects of symmetries are shown up for separated $m$ manifolds only (Fig. 3b).

For non-interacting electrons $(\lambda=0)$, the energy levels of the QD are Fock-Darwin levels [11]

$$
\epsilon=\tilde{\omega}_{\rho}\left(2 n_{\rho}+|m|+1\right)+\tilde{\omega}_{z}\left(n_{z}+\frac{1}{2}\right)-\tilde{\omega}_{L} m .
$$

For rational ratios of $\omega_{z} / \omega_{\rho}$ the energy levels Eq. (9) are degenerate. It is simply the spectrum of the RHO in the external field $\tilde{\omega}_{L} m$. For instance, at $\omega_{z} / \omega_{\rho}=2$ we have $\epsilon=\tilde{\omega}_{z}(N+|m| / 2+1)-\tilde{\omega}_{L}^{\prime \prime} m$. The quantum number $N=n_{\rho}+n_{z}=n_{1}+n_{2}=0,1,2, \ldots$ and each $m$-manifold consists of the shells characterized by this quantum number. Since the eigenenergies of the term $H_{\mathrm{CM}}$ with the corresponding quantum numbers are determined by Eq. (9) as well, the shells in the total spectrum of the QD are not affected.

The Coulomb interaction destroys the general symmetry of the 3D HO. However, the magnetic field can recover symmetries which are common for the RHO and Coulomb systems. At a relatively low value of the magnetic field $\omega_{L}^{\prime \prime}$ (for our parameters $B \approx 2.4 \mathrm{~T}$ ) we reveal the first manifestation of the hidden symmetries. This symmetry is determined by the integral of motion, Eq. (4). It results in the appearance of shells at each $m$ manifold (Fig. 3b). There are exact crossings and repulsions between levels of different and of the same parity, 
respectively, in each shell. The near-degeneracy of the quantum spectrum is reminiscent of a striking degeneracy observed for the RHO or pure Coulomb systems. At higher values of the magnetic field $\omega_{L}^{\prime}(B \approx 7.5 \mathrm{~T})$, the dynamical spherical symmetry appears, since $\mathbf{l}^{2}$ becomes an additional integral of motion. This symmetry manifests itself as the attraction between levels with different orbital quantum numbers and the same parity (Fig. 3b). In contrast to spectra of pure Coulomb systems or of the RHO, there are no crossings between eigenstates of the subset characterized by a given quantum number $m$, since the accidental degeneracy is removed. Although the symmetry is recovered at very strong magnetic field $\omega_{L}^{\prime \prime \prime}(B \approx 15.9 \mathrm{~T})$ due to the appearance of the integral of motion Eq. (7), the dynamics is non-separable for $m \neq 0$. Note that shells are similar to the spherical case.

The symmetries may be detected by studying the conductance of two-electron QDs at low temperatures. In particular, at $\omega_{L}^{\prime \prime}$ in the excited states there is the onset of a singlet-triplet degeneracy related to crossings of the eigenstates (6) with $|c|>0$ (see Fig. 3b). The total spin $S$ alternates between 1 and 0 and the addition of a second electron with a spin-up or spin-down orientation to the QD will cost the same energy. At zero magnetic field $B=0$ two electrons occupy the same state with $S=0$. At $\omega_{L}^{\prime \prime}$ it becomes favorable for one electron to flip its spin. The electron reconfigures the charge and polarizes the two-electron QD leading to a Kondo type effect [14], i.e., to the increase of the conductance at low temperatures. The enhancement of the conductance in QDs due to the singlet-triplet degeneracy induced by the magnetic field have been discussed in [15-17] (for review see [18]). According to Ref. [18], when the system is tuned to the degeneracy point the differential conductance, $d I / d V$, would exhibit a peak at zero bias. The increase of the strength of the magnetic field removes the degeneracy and the peak will split onto two peaks reflecting the single-particle spacing between singlet-triplet states. It should be noted, however, that this prediction is obtained in Ref. [18] within a schematic model where single-particle levels and the magnetic field are adjustable parameters. In addition, the electron-electron interaction is assumed to be weak. According to our analysis the onset of the singlet-triplet degeneracy holds at any strength of the electron-electron interaction in QDs. We suggest the mechanism, related to the hidden symmetries, responsible for the occurrence of this degeneracy in two-electron 3D QDs at a certain value of the magnetic field.

Summarizing, we have shown that the axially symmetric $3 \mathrm{D}$ quantum dot with two electrons exhibits hidden symmetries at certain values of the magnetic field. In particular, due to these symmetries the onset of a singlettriplet degeneracy in excited states is found when the magnetic field value is $\omega_{L}^{\prime \prime}$. Finally, we are thankful to Jan-M. Rost for valuable discussions and constructive suggestions.

[1] L.D. Landau and E.M. Lifshitz, Quantum Mechanics (Oxford, Pergamon Press, 1977).

[2] P.A. Maksym, H. Imamura, G.P. Mallon and H. Aoki, J.Phys.: Condens. Matter 12, R299 (2000).

[3] L.P. Kouwenhoven, D.G. Austing, and T. Tarucha, Rep. Prog. Phys. 64, 701 (2001).

[4] R.C. Ashoori, Nature (London) 379, 413 (1996).

[5] J.M. Jauch and E.L. Hill, Phys. Rev. 57, 641 (1940).

[6] R. Blümel, C. Kappler, W. Quint, and H. Walther, Phys. Rev. A 40, 808 (1989).

[7] Y. Alhassid, E.A. Hinds, and D.Meschede, Phys. Rev. 
Lett. 59, 1545 (1987).

[8] R.G. Nazmitdinov, N.S. Simonović, and J.M. Rost, Phys. Rev. B 65, 155307 (2002).

[9] R.C. Ashoori, H.L. Stormer, J.S. Weiner, L.N. Pfeiffer, K.W. Baldwin and K.W. West, Phys. Rev. Lett. 71, 613 (1993).

[10] M. Dineykhan and R.G. Nazmitdinov, Phys. Rev.B 55, 13707 (1997).

[11] V. Fock, Z. Phys. 47, 446 (1928); C.G. Darwin, Proc. Cambridge Philos. Soc. 27, 86 (1930).

[12] L. Brey, N. Johnson, and B.I. Halperin, Phys. Rev. B 40, 10647 (1989); Q.P. Li, K. Karraï, S.K. Yip, S. Das Sarma, and H.D. Drew, Phys. Rev. B 43, 5151 (1991).

[13] I.V. Komarov, L.I. Ponomarev, and S.Yu. Slavyanov, Spheroidal and Coulomb Spheroidal Functions (in Russian) (Nauka, Moscow, 1976).

[14] D. Goldhaber-Gordon, H. Shtrikman, D. Mahalu, D. Abusch-Magder, U. Meirav, and M.A. Kastner, Nature (London) 391, 156 (1998); J. Schmid, J. Weis, K. Eberl, and K.v. Klitzing Phys.Rev.Lett. 84, 5824 (2000); S. Sasaki, S. De Franceschl, J.M. Elzerman, W.G. van der Wiel, M. Eto, S. Tarucha, and L.P. Kouwenhoven, Nature (London) 405, 764 (2000).

[15] Y. Meir, N.S. Wingreen, and P.A. Lee, Phys.Rev.Lett. 70, 2601 (1993).

[16] M. Pustilnik, Y. Avishai, and K. Kikoin, Phys.Rev.Lett. 84, 1756 (2000).

[17] M. Pustilnik and L.I. Glazman, Phys.Rev.Lett. 85, 2993 (2000).

[18] M. Pustilnik, L.I. Glazman , D.H. Cobden and L.P. Kouwenhoven, Lecture Notes in Physics, 579, 3 (Springer, Berlin, 2001).

\section{Figure Captions}

FIG. 1. Poincaré surfaces of sections $z=0, p_{z}>0$ of the relative motion $(\lambda=3, \epsilon=10, m=0)$ with: (a) $\omega_{z} / \omega_{\rho}=5 / 2$, (b) $\omega_{z} / \omega_{\rho}=2$ and (c) $\omega_{z} / \omega_{\rho}=3 / 2$. The section (b) indicates that for the ratio $\omega_{z} / \omega_{\rho}=2$ the system is integrable.

FIG. 2. Typical trajectories $(\epsilon=10, m=1)$ of the relative motion at $\lambda=3$ for $\omega_{z} / \omega_{\rho}=1(\mathrm{a}, \mathrm{b})$ and $\omega_{z} / \omega_{\rho}=2(\mathrm{c}, \mathrm{d})$ are shown in cylindrical and prolate spheroidal coordinates, respectively.

FIG. 3. The lowest eigenenergies of Hamiltonian (2) (in units $\left.\hbar \omega_{0}\right)$ at $\omega_{z} / \omega_{0}=2.5$ and $\lambda=3$ as functions of the ratio $\omega_{L} / \omega_{0}$ for: (a) all $m$; (b) $m=0$. The upper energy limit is chosen high enough to amplify the shell structure of the spectrum. The integrable cases: $\omega_{z} / \omega_{\rho}=2,1$ and $1 / 2$ are indicated by vertical dotted lines $\left(\omega_{L} / \omega_{0}=0.75, \sqrt{21} / 2\right.$ and $\sqrt{24}$, respectively). 
This figure "fig1.gif" is available in "gif" format from: http://arxiv.org/ps/cond-mat/0211631v1 
This figure "fig2.gif" is available in "gif" format from: http://arxiv.org/ps/cond-mat/0211631v1 
This figure "fig3.gif" is available in "gif" format from: http://arxiv.org/ps/cond-mat/0211631v1 